\title{
Changing patterns of cell surface mono (ADP-ribosyl) transferase antigen ART2.2 on resting versus cytopathically-activated T cells in NOD/Lt mice
}

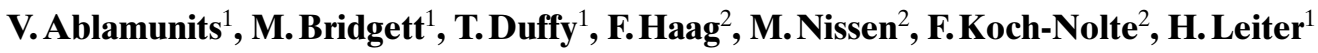 \\ ${ }^{1}$ The Jackson Laboratory, Bar Harbor, Maine, USA \\ ${ }^{2}$ Institute for Immunology, University Hospital, Hamburg, Germany
}

\section{Abstract}

Aims/hypothesis. ART2.2 is a mouse T-cell surface ectoenzyme [mono (ADP-ribosyl) transferase] shed upon strong activation. We analysed temporal changes in ART2.2 expression in unmanipulated and cyclophosphamide-treated NOD/Lt mice compared with diabetes-resistant control strains. We used NAD, the ART2.2 substrate, to test whether ARTmediated ADP-ribosylation could retard diabetogenic activation of islet-reactive T cells in vitro.

Methods. ART2.2 and CD38, another NAD-utilizing enzyme, were measured by flow cytometry. ADP-ribosylation from ethano-NAD was followed by flow cytometry using a reagent specific for etheno-ADP ribose.

Results. Although mature NOD CD $4+$ and C D $8+\mathrm{T}$ cells expressed ART2.2, this expression was delayed in young NOD mice when compared with control strains. This ontological delay at 3 weeks of age cor- related with an early burst of CD25 expression unique to NOD splenic T cells. This pattern was reproduced in cyclophosphamide-accelerated diabetes in young NOD/Lt males, wherein a retarded repopulation of ART2.2 T cells in spleen and islets correlated with development of heavy insulitis and diabetes. NAD inhibited anti-CD3 induced activation of splenic T cells in vitro and also retarded killing of beta-cell targets by NOD islet-reactive CD8 effectors in vitro at concentrations equal to or greater than $1 \mu \mathrm{mol} / 1$. Evidence suggested that CD38 on B lymphocytes competes with ART2.2 for substrate needed by B lymphocytes for ADP ribosylation.

Conclusions. ART2.2 on T cells may not simply mark the resting state, but could also contribute to it via ADP-ribosylation. [Diabetologia (2001) 44: 848-858]

Keywords Type I diabetes, NOD mice, T lymphocytes, cell surface molecules, ADP-ribosyl transferase, CD38.
Received: 27 November 2000 and in revised form: 1 March 2001

Corresponding author: Dr. Edward H. Leiter, The Jackson Laboratory, 600 Main St., Bar Harbor, ME 04609 USA, email: ehl@jax.org

Abbreviations: ART, mono (ADP-ribosyl) transferase; B6, C57BL/6 J; BKS,C57BLKS/J; BB, BioBreeding; Cal, calcium ionophore A23187; CML, cell-mediated lysis; CTL; cytotoxic $\mathrm{T}$ cell; CYP, cyclophosphamide; DAB, diaminobenzidine; IL2R, interleukin 2 receptor; $\mathrm{mAb}$, monoclonal antibody; NOD, non-obese diabetic; PARP, poly (ADP-ribose) polymerase; FITC, fluorescein isothiocyanate; PE, phycoerythrin; APC, allo-phycocyanin; TCR, T cell receptor; Tg, transgenic.
Mouse Chromosome 7 contains a cluster of genes encoding functional mono ADP-ribosyltransferases (ART) expressed on the surface of T cells [1]. These enzymes are capable of catalyzing the hydrolysis of nicotine adenine dinucleotide (NAD) into ADP-ribose and nicotinamide [2], and the transfer of the ADP-ribose moiety onto arginine residues of T-cell surface molecules associated with transmission of activation signals or with adherence [3]. One of these genes, designated Art1, was initially cloned from YAC1 cells and represents a mouse ortholog of a gene cloned from rabbit skeletal muscle [4]. A tandem duplication of another mouse Art gene tightly linked to $A r t 1$ and now designated Art $2 a$ and $A r t 2 b$ represents mouse orthologs of the single rat gene en- 
coding the RT6 alloantigen $[2,5]$. In both genera, ART2/RT6 is a differentiation antigen expressed on a small percentage of the most mature thymocytes and on resting peripheral T cells [6].

Studies correlating the immunoregulatory potential of $\mathrm{T}$ cells in lymphopenic and non-lymphopenic stocks of BB rats with RT6 expression suggest that ARTs could mediate important immunoregulatory functions in the control of autoimmune, T-cell mediated Type I (insulin-dependent) diabetes mellitus [7]. In peripheral T cells of most rat strains, the molecule is expressed on approximately $70 \% \mathrm{CD} 8+$ and $50 \%$ CD 4 + cells [7]. T-lymphopenia in diabetesprone $\mathrm{BB}$ rats, however, is characterized by a dearth of ART2/RT6- positive T cells. Diabetes-resistant BB-DR/Wor rats have normal numbers of circulating ART2-positive T cells but will become diabetic after monoclonal antibody (mAb) depletion of ART2/ RT6-positive T cells coupled with stimulation of the ART-negative population with Kilham rat virus or poly I:C challenge [8]. Co-transfer of ART2/RT6-positive $\mathrm{T}$ cells from non-lymphopenic BB-DR rats prevents adoptive transfer of Type I diabetes by ART2/ RT6-depleted DR-derived T cells into athymic MHC compatible recipients [9].

Mouse T cells stimulated in vitro in the presence of NAD, the ART substrate, have reduced proliferation, IL2 secretion, and killing function. This inhibition was associated with ADP-ribosylation of LFA1, CD8, CD27, and CD43-45 [10]. Type I diabetes-prone NOD mice differ from T-lymphopenic BB rats in that excessive numbers of mature non-obese diabetic (NOD) T-cells emigrate from the thymus and stably populate the periphery ("T-lymphoaccumulation"). Initial northern blot analysis of ART2 mRNA concentrations in NOD/Lt showed that although the genes were expressed in the early post-weaning period, lower mRNA concentrations were observed in $\mathrm{T}$ cell-enriched NOD/Lt spleens as compared to the related NON/Lt strain [11]. Recently, gene gun immunization of rats with $A r t 2 b$ cDNA generated a panel of $\mathrm{mAb}$ against the protein product designated ART2.2 (the product of the Art2 $a$ locus is ART2.1, but no $\mathrm{mAb}$ have been generated yet). Using one of these mAb, Nika 102, we found that ART2 marked a small number $(1.5 \%)$ of the most mature CD4 or CD8 single positive thymocytes [6]. Using flow cytometry to assess ART2.2 expression on T cells from adult NOD/ Lt mice, we found that 30 to $40 \%$ of splenic CD 4 + cells and 70 to $90 \%$ of CD 8 + cells expressed this antigen on their surfaces, comparable to percentages observed in non-Type I diabetes-prone C57BL/6 $\mathrm{J}$ and C57BLKS/J strains [6]. This antigen apparently marked resting $\mathrm{T}$ cells in the periphery, since activation elicited rapid metalloprotease-mediated shedding with the same kinetics exhibited by another surface marker, L-selectin $[6,12]$. This shedding was inversely correlated with induction of CD25 (IL2R) ex- pression [6]. Islet autoreactive NOD $\mathrm{T}$ cells shed ART2.2 within $2 \mathrm{~h}$ after T cell receptor cross-linking or activation of protein kinase $\mathrm{C}$ by phorbol ester [12]. Activated T cells that had shed ART2.2 were markedly less sensitive to ADP-ribosylation.

In this study, we report the expression of the ART2.2 antigen on splenic, peripheral blood and islet-infiltrating T cells in the natural history Type I diabetes development and after acceleration of the disease by cyclophosphamide (CYP). We show that cytolytic function of NOD islet-reactive cytoxic T cells (CTL) can be diminished by pretreatment with NAD at concentrations as low as $1 \mu \mathrm{mol} / \mathrm{l}$. We further compare ART2.2 expression to that of CD38, a ubiquitously-expressed NAD catabolizing enzyme that produces nicotinamide, cyclic ADP-ribose (cADPR), and ADP-ribose. Unlike ART2.2, CD38 is not shed upon T-cell activation and could represent a strong competitor of ART for binding to the NAD substrate [13]. The results to be presented suggest that ART ectoenzymatic activity could potentially be used as a means of controlling the activation and cytotoxic function of islet-reactive T cells.

\section{Materials and methods}

Mice. NOD/Lt, NON/Lt, NOR/Lt, NOD/LtSZ.CB17 Prkd $^{\text {scid }}$ ) (denoted here as NOD.scid), and C57BLKS/J (BKS) mice were bred in our research colony at The Jackson Laboratory (TJL). C57BL/6 J (B6), CBA/J, BALB/cByJ, and $\mathrm{NZW} /$ LacJ mice were obtained from the Animal Resources Unit of TJL. A stock of NOD mice doubly transgenic $(\mathrm{Tg})$ for the rearranged $\mathrm{V} \alpha 8 \mathrm{~V} \beta 2 \mathrm{~T}$-cell receptor (TCR) of a diabetogenic CD8 + T-cell clone (here designated NOD.AI4 $\alpha \beta \mathrm{Tg}$ ) were kindly provided by Dr. D. Serreze at TJL and are described elsewhere [14].

Antibodies. Rat anti-mouse ART2.2 monoclonal antibody (Nika 102) was purified from hybridoma culture supernatants and was used for immunohistochemistry (unlabelled) and flow cytometry (FITC-conjugated). Mouse anti-etheno-NAD monoclonal antibody $1 \mathrm{G} 4$ [15], kindly provided by Dr. R. Santella, Columbia University, New York, N. Y., USA, was used to assess the degree of T-cell surface mono-ADP ribosylation [12]. Goat anti-mouse Ig antibody conjugated to fluorescein isothiocyanate (FITC) or phycoerythrin (PE) were from Fisher Scientific, (Pittsburgh, Pa., USA). Fluorochrome-conjugated anti-mouse CD3e (145-2C11-FITC), CD8 [53-6.72 conjugated with phycoerythrin (PE) or allo-phycocyanin(APC)], CD25(7D4-FITC), CD19 (1D3-FITC) and CD38 (90-PE) were purchased from Pharmingen (San Diego, Calif., USA). GK1.5 (anti-CD4) was conjugated with Cy3 using a commercial kit (FluoroLink Cy3 Reactive Dye; Amersham, Piscataway, NJ., USA) or purchased as an APC conjugate from PharMingen. Hamster anti-mouse $\mathrm{CD} 3 \varepsilon$ was purified from 145-2C11 hybridoma supernatant (ATCC, Rockville, Md., USA) and was used at $2 \mu \mathrm{g} / \mathrm{ml}$ for T-cell activation in vitro. Rat anti-mouse CD45R/B220 (clone RA3-6B2) was purified from culture supernatant and used for B-lymphocyte depletion by BioMag magnetic beads coated with goat anti-rat IgG (PerSeptive Diagnostics, Cambridge, Mass., USA) 
Isolation of splenic, peripheral blood, and islet lymphocytes. At the indicated timepoints, mice were killed by cervical dislocation and spleens were aseptically removed and meshed through a Nitex sieve (Tetko, Kansas City, Mo., USA). Red blood cells were lysed and the nucleated cells washed and counted. For B-lymphocytes depletion, spleen cells were resuspended at $4 \cdot 10^{7}$ per $0.5 \mathrm{ml} \mathrm{PBS}$ and incubated on ice with $50 \mu \mathrm{g}$ anti-B220 mAb for $45 \mathrm{~min}$. Then the cells were washed, incubated with BioMag goat anti-rat IgG suspension and separated on the BioMag separator (Advanced Magnetics, Cambridge, Mass., USA) according to the manufacturer's instructions. Blood was obtained by cardiac puncture. For islet-infiltrating lymphocytes, pancreatic islets were isolated by the intraductal collagenase technique as previously described [16] and dispersed by gentle pipeting into a single cell suspension in non-enzymatic dissociation buffer (Sigma, St. Louis, Mo., USA).

Cyclophosphamide treatment. Cyclophosphamide (CYP, Sigma) was dissolved in PBS (Life Technologies, Rockville, Md., USA) and administered i.p to 10-week-old males in a single dose of $250 \mathrm{mg} / \mathrm{kg} \mathrm{b}$ w. Pancreata for histological examination were removed at days $0,3,5$ and 8 . Spleens and peripheral blood were analysed by flow cytometry at days $0,3,5,8,14$ and 22 .

Flow cytometrie. Cells $\left(10^{6}\right)$ were stained with pre-titrated antibodies in PBS containing $1 \%$ BSA and $0.05 \%$ sodium azide at $4{ }^{\circ} \mathrm{C}$ for $30 \mathrm{~min}$, washed and analysed on the FACScan (Becton Dickinson, Mountain View, Calif., USA). Dead cells were excluded by propidium iodide gating. Per cent positive cells were calculated using CellQuest software. Multichannel flow cytometry was used to compare the levels of CD25 and ART2.2 expression on $\mathrm{T}$ cells from 3 to 7-week-old mice (FACS caliber flow cytometer. Becton Dickinson, Mountain View, Calif., USA): Samples were stained with anti CD25-PE conjugate, anti ART2.2-FITC, and anti-CD4 and anti-CD8 (the latter two reagents both conjugated to APC). The anti CD8-APC conjugate was used at a more dilute concentration to resolve the $\mathrm{CD}^{+}$population between the unstained and $\mathrm{CD} 4^{+}$-APC populations. To obtain the correct dilutions of these antibodies each was titrated using NOD spleen and plotted in histogram format using CellQuest. The dilutions of the antibody lot used in this experiment were 1:128 for the anti CD4-APC conjugate and 1:600 for the anti CD8-APC conjugate with the anti-CD8 having the lower mean fluorescence.

Histology. For immunohistochemical evaluation of ART2.2 in the pancreas, snap-frozen pancreata were sectioned at $8 \mu \mathrm{m}$ thickness, acetone-fixed (10 min at RT), air-dried, and washed with PBS. Endogenous peroxidase was blocked using a peroxidase blocking reagent (DAKO Carpinteria, Calif., USA) for 10 min. A commercial kit using biotinylated rabbit anti-rat $\mathrm{Ab}$ and streptavidin-HRP with DAB development was used according to manufacturer's instructions for immunodetection of Nika 102 binding (rat ABC kit, Vector Laboratories, Burlingame, Calif., USA). Non-specific staining was blocked using normal rabbit serum, and controls omitting the primary antibody were included. Primary antibodies were added [either Nika $102(5 \mu \mathrm{g} / \mathrm{ml})$ or irrelevant AA4.1 (for negative IgG2b isotype-matched control)] and slides incubated overnight at $4{ }^{\circ} \mathrm{C}$. Slides were counterstained with Mayer's haematoxylin and mounted. For evaluation of insulitis and insulin content, Bonin's fixed paraffin-embedded pancreatic sections (3 nonoverlapping sections at $300 \mu \mathrm{m}$ intervals, containing $25-40$ islets/specimen) were stained with aldehyde fuchsin and counterstained with haematoxylin-eosin. The scoring system for in- sulitis was 0 for no islet-associated lymphocytes, 1 for peri-insulitis, 2 for penetrating infiltrate affecting less than $30 \%$ of the islet-area, 3 for insulitis affecting more than $30 \%$ of the islet area, and 4 for end-stage islets with few or no granulated beta cells.

Effects of NAD in vitro. To study the effects of NAD on polyclonal T-cell activation, spleen cells were cultured in MEM alpha medium containing Glutamax and gentamycin (Life Technologies) and $10 \%$ FCS (HyClone, Logan, Utah, USA) with or without anti-CD3 Ab. Nicotine adenine dinucleotide was dissolved in complete medium at final concentrations of 0.1 to $100 \mu \mathrm{mol} / \mathrm{l}$. After incubation for $18 \mathrm{~h}$, the cells were washed and triple-stained for CD4, CD8 and CD25 and analysed by flow cytometry. To test the influence of NAD on T cell-mediated killing of islet cells, $5 \cdot 10^{5}$ spleen cells from NOD.AI $\alpha \beta$ Tg mice [14] were added to $10^{4} \mathrm{Cr}^{51}$-labelled NOD.scid islet cells for $18 \mathrm{~h}$ in the presence of $0.1-10 \mu \mathrm{M}$ NAD. Supernatant radioactivity was measured using the Wallac Wizard 1470 gamma counter (Wallac Oy, Turku, Finland), and per cent specific lysis was calculated using the formula:

$\%$ specific lysis $=100 \cdot\left(\mathrm{cpm}_{\exp }-\mathrm{cpm}_{\text {spont }}\right) /\left(\mathrm{cpm}_{\text {total }} \mathrm{cpm}_{\text {spont }}\right)$,

where $\mathrm{cpm}_{\text {spont }}$ is $\mathrm{Cr}^{51}$ release in the absence of spleen cells and $\mathrm{cpm}_{\text {total }}$ is release in the presence of $10 \%$ SDS.

Mono-ADP ribosylation of $T$ cells. Whole spleen cells or $\mathrm{B}$ lymphocyte-depleted preparations (87-92\% T cells) were incubated with $100 \mu \mathrm{mol} / \mathrm{l}$ etheno-NAD (Sigma) for $20 \mathrm{~min}$ at $37^{\circ} \mathrm{C}$ in MEM alpha medium, washed, stained with $1 \mathrm{G} 4 \mathrm{mAb}$ followed by goat anti-mouse Ig-FITC or PE and analysed by FACS. To study the effects of T-cell activation on ADP-ribosylation, cells were from B lymphocyte-depleted preparations were incubated for $2 \mathrm{~h}$ with PMA $(10 \mathrm{ng} / \mathrm{ml})$ and calcium ionophore A23187 (CaI, $500 \mathrm{ng} / \mathrm{ml}$ ) (both from Sigma) and washed before adding etheno-NAD.

Statistics. Significance of differences was assumed at a $p$ value of less than 0.05 by a Student's $t$ test (two-tailed) or Wilcoxon signed rank test.

\section{Results}

Delayed ontogeny of ART2.2 expression in NOD mice. We have previously reported that peripheral $\mathrm{T}$ cells of adult NOD/Lt mice, unlike those of BB-DP rats, contain numerous ART2.2-positive cell of both CD4 and CD8 subset [6]. However, this finding was at variance with our earlier finding of low ART mRNA concentrations in spleen of young NOD/Lt mice [11]. To assess whether NOD/Lt mice had strain-specific anomalies in developmental regulation of the ART2.2 ectoenzyme, we compared the ontogeny of ART2.2 antigen expression on splenic T cells from NOD mice with the related, but Type I diabetes-resistant NOR/Lt and NON/Lt strains, as well as the NOR/Lt-related BKS strain. As shown in Figure 1 expression of ART2.2 in both T-cell subsets of all strains showed an age-dependent increase. However, NOD females maintained much higher numbers of ART2.2-negative CD4 and CD8 T cells over the peripubertal period when compared to the related control rats (NON/Lt and NOR/Lt) and also when com- 

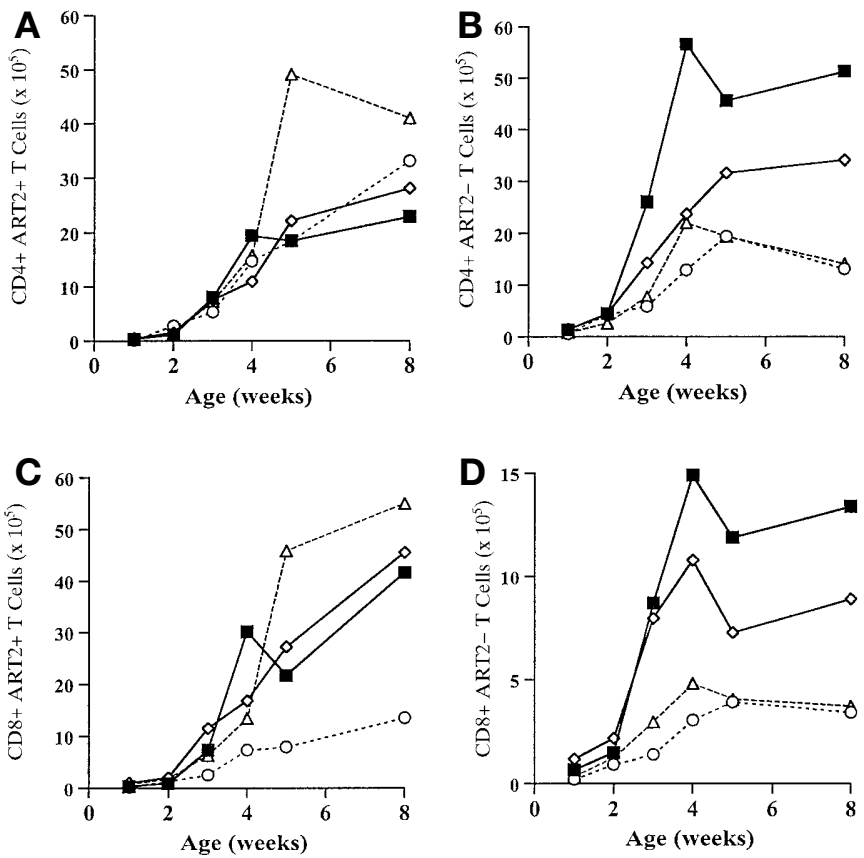

Fig. 1. Ontogeny of ART2.2 expression on splenic T cells from NOD and related strains. Spleen cells from three mice of each strain per time point were stained for CD4, CD8, and ART2.2 and analysed by flow cytometry [NOD $(\mathbf{\square})$, BKS $(\triangle)$, NOR $(\diamond)$, NON $(\bigcirc)$. Data for C57BLKS $(\mathrm{BKS})$ mice are shown for comparison, because NOR mice contain approximately $12 \%$ of the BKS genome, including the Art $2 b$-containing region on Chr.7. Data shown are means. Standard errors were too small to show on the plot. Wilcoxon signed rank test resulted in $p<0.03$ for NOD compared to the other strains

pared to BKS, a parental contributor to the NOR/Lt strain. This reduced proportion of ART2.2-positive T cells in spleens of young NOD/Lt females was particularly striking when compared with $\mathrm{NON} / \mathrm{Lt}$ spleens. NON/Lt mice have markedly lower total numbers of CD4 and CD8 splenic T cells than are found in age-matched NOD/Lt spleens, yet a higher percentage of both subsets in NON/Lt were ART2.2-positive by 3 weeks of age. At the 3 week timepoint, $48 \%$ of CD4-positive T cells in NON/Lt were ART2.2-positive as compared to $24 \%$ in NOD/ Lt. Similarly, $64 \%$ of the NON/Lt CD8-positive fraction were ART2.2-positive, verus $46 \%$ in NOD/Lt. By 12 weeks of age, percentages of splenic CD4/ ART2.2 and CD8/ART2.2 double-positive NOD T cells had increased to percentages comparable to the other strains (data not shown).

The transiently reduced percentages of ART2.2positive splenic T cells in NOD mice during the early peripubertal period were accompanied by a NOD strain-specific upregulation of CD25 (IL2 receptor B-chain) when ART2.2 percentages were reduced (Fig. 2). This is the age at which the first accumulation of lymphocytes in islets is reported $[17,18]$. Four color staining of NOD/Lt T cells showed that it was pre-

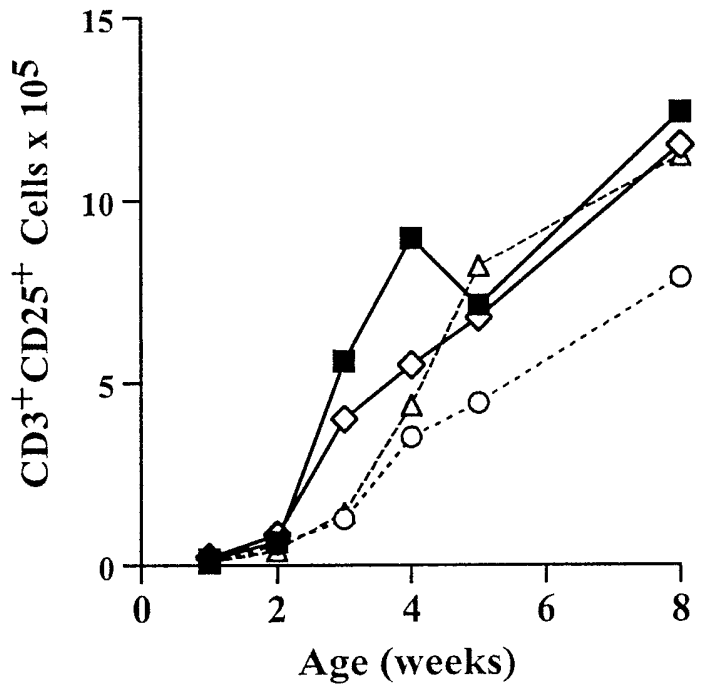

Fig. 2. Ontogeny of CD25 on splenic T cells from NOD and related strains. Spleen cells from three mice of each strain per time point were stained for CD3 and CD25 and analysed by flow cytometry. A peak of CD25 staining seen on the NOD T cells at week 3 was reproduced in a separate experiment. Data shown are means. $* * p<0.01$ for NOD vs NOR and NON. $* p<0.05$ for NOD vs NOR and NON. [NOD (ם), $\operatorname{BKS}(\triangle), \operatorname{NOR}(\diamond), \operatorname{NON}(\bigcirc)]$

dominantly the CD4-positive T cells ( $>90 \%$ ) that were expressing CD25. At the 4 week time point, $15 \%$ of the total CD4-positive population expressed CD25. The majority of these $(78 \%)$ were ART2.2negative.

Differential expression of ART2.2 in the periphery versus in islet infiltrates. In organ-specific autoimmune diseases like Type I diabetes, T cells in the insulitis lesion would be presumed to contain a higher proportion of activated cells than in spleen or peripheral blood because the former are immediately involved in the autoimmune destruction. To examine whether reduced ART2.2 expression might be a surrogate marker for tracking autoreactive $\mathrm{T}$ cells in NOD, we did a longitudinal analysis of ART2.2 expression on $\mathrm{T}$ cells from spleen, peripheral blood, and islet-associated lymphocytes. The per cent of ART2.2-positive $\mathrm{T}$ cells was highest in the spleen (Fig.3). A lower percentage was observed in blood, and a considerably smaller and highly-variable percentage of ART2.2-positive T cells was isolated from islets (Fig. 3).

Cyclophosphamide-induced loss of ART2.2-positive T cells correlates with accelerated diabetogenesis. Further indication that a fall in ART2.2 expression might presage cytopathic activation of T-effectors was provided by CYP-accelerated diabetogenesis in young NOD males. If the transition of a T-effector cell from a resting to an activated state entails shedding 


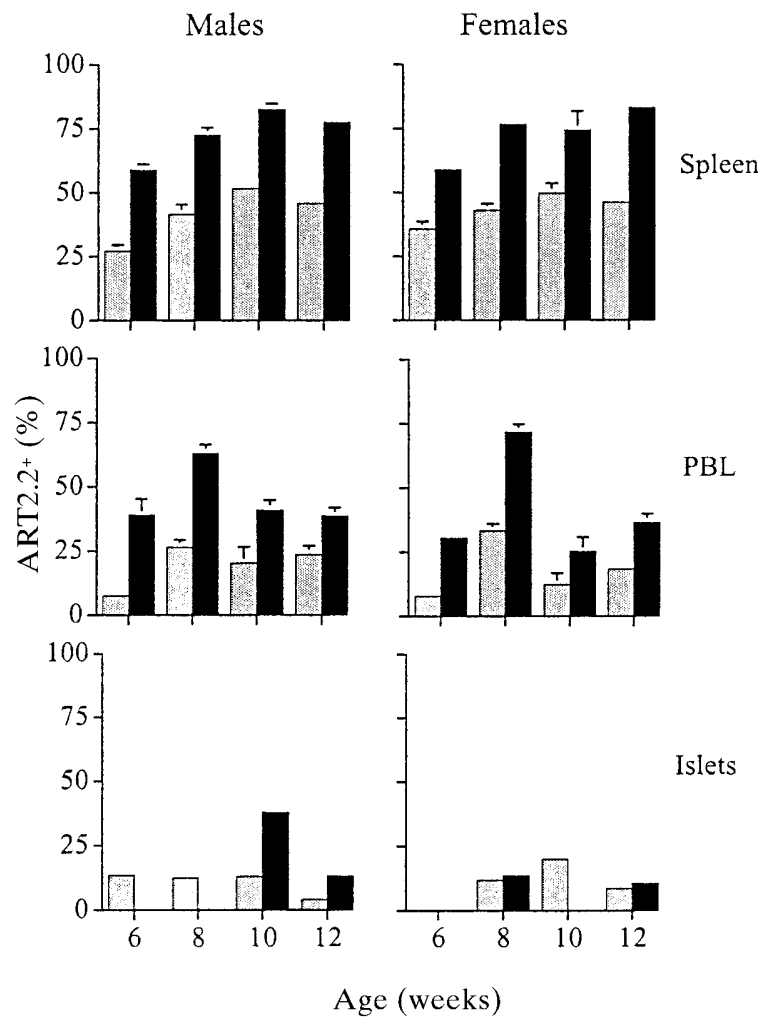

Fig.3. ART2.2 expressions on $\mathrm{T}$ cells from different anatomic compartments of pre-diabetic NOD mice. Splenic and PBL from three mice per sex and age group were stained and analysed. Islet-infiltrating lymphocytes had to be pooled from a minimum of five mice per donor group due to low cell yields. Data are means \pm SE for splenocytes and PBL, while islet lymphocyte data are from one serial experiment. Absence of data points in the lower panels indicate that the per cent of ART2.2-positive islet-infiltrating $\mathrm{T}$ cells was not calculated due to the low number of total events $(<100) . p<0.01$ for $\mathrm{CD}^{+}(\square)$ splenocytes and $p<0.002$ for $\mathrm{CD}^{+}(\square)$ splenocytes vs respective PBL (Mann-Whitney two-tailed test). Difference between males and females was NS

(or non-expression) of the ART2.2 ectoenzyme, then it would be anticipated that reduced ART2.2 expression should correlate with CYP-accelerated diabetogenesis. Cyclophosphamide induced a severe transient lymphodepletion seen early after its administration, which was followed by a rapid repopulation of the spleen (Fig. 4A), so that the absolute T-cell numbers were restored by day 8 after a single injection. However, the absolute numbers of ART2.2-positive CD4 and CD8 T cells in spleen remained low even until the day 15 timepoint (Fig. 4, B-C), indicating that the repopulating spleen contained an activated population of ART2.2-negative T cells. During this period of markedly reduced ART antigen expression, a progressive increase in the severity of insulitis was observed in the CYP-treated group compared with the controls (Fig. 4, D). In the group of three males aged to the 21-day timepoint, two had developed glycosuria. FACS analysis of ART2.2 + T cells associat-

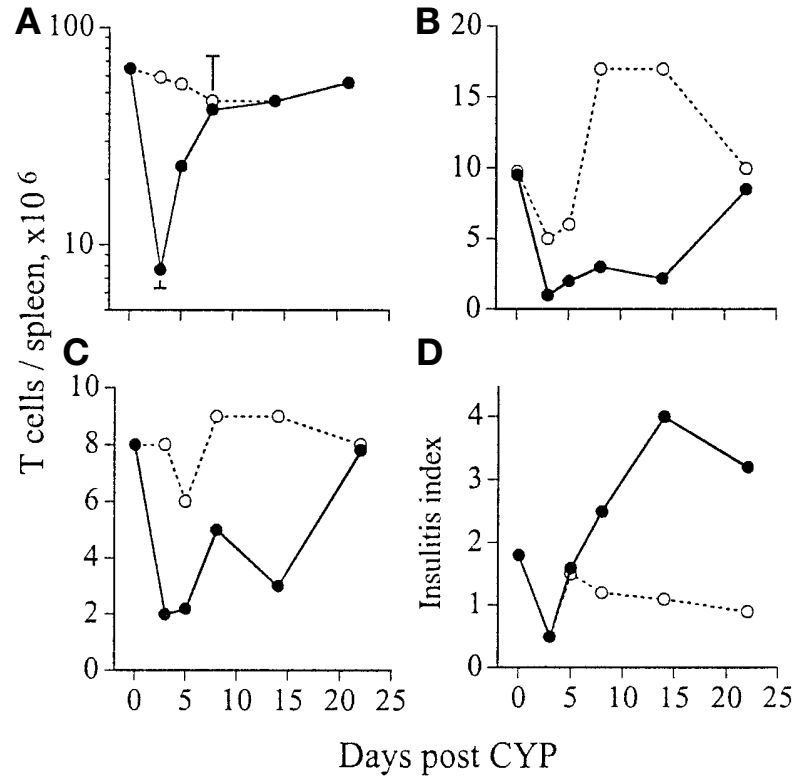

Fig.4A-D. Delayed ontogeny ART2 2.expression in CYP-accelerated diabetes. NOD males, 10 weeks of age, were treated with CYT (--) or PBS (-- - --) as described in Materials and methods. Three mice at each time point were killed and spleen cells counted and stained for CD4, CD8, and ART2.2. The absolute numbers of ART2.2-positive CD4 and CD8 $\mathrm{T}$ cells were calculated. (A) Total numbers of $\mathrm{T}$ cells per spleen; (B) Numbers of ART2.2-positive CD4 T cells; (C) Numbers of ART2.2-positive CD8 T cells (D) Mean severity of insulitis assessed by histological examination of the pancreata. Data are means of three mice per time point from one of two replicative experiments

ed with islets of PBS-treated controls at the day 5 timepoint indicated $23.8 \%$ positive cells versus only $1.5 \%$ positive in T cells from CYP-treated males. Because total numbers of ART2.2-positive islet-infiltrating lymphocytes estimated by FACS analysis were low even in unmanipulated mice, we studied the effects of CYP on ART2.2 expression in the lesion by immunohistochemistry. As shown in Figure 5, CYP administration was associated with a dramatic loss of ART2.2-positive T cells in the islet infiltrate at day 5 after CYP treatment, the intensity of staining slowly increasing only from day 8 onward. This down-regulation of ART2.2 expression in the spleen and in the pancreas could reflect increased numbers of activated $\mathrm{T}$ cells due to a more efficient antigen presentation or it could result from a preferential depletion of regulatory T cells by CYP, as suggested previously [20].

T-cell activation and cytotoxicity in vitro is inhibited by NAD. The high proportion of ART2.2-positive T cells in spleens of unmanipulated NOD adults raises the question of whether ART2.2 enzymatic properties (e.g., ADP-ribosylation) can modulate immune responses. We first assessed differences in CD25 upregulation in an in vitro system wherein splenic $\mathrm{T}$ 

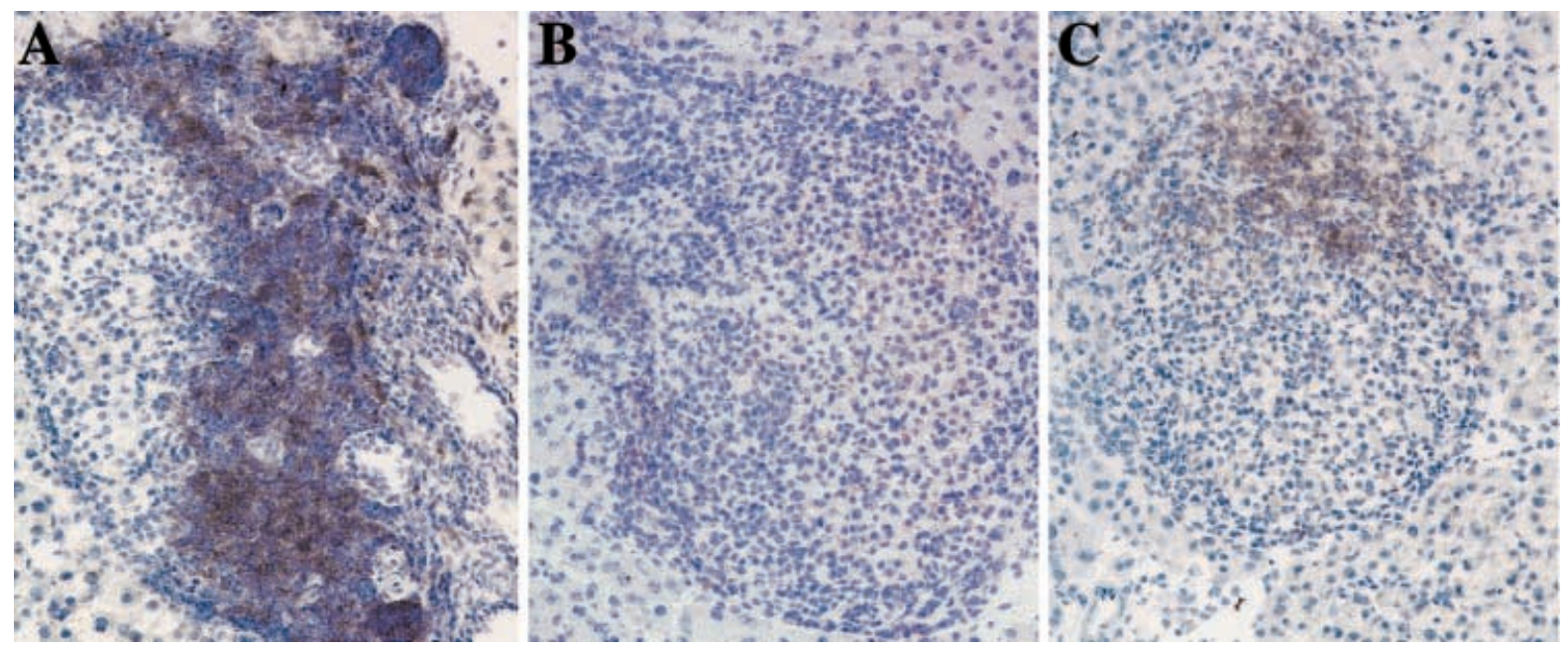

Fig.5A-C. Reduced accumulation of ART2.2-positive T cells in the CYP-accelerated NOD insulitic lesion. ART2.2 antigen was immunocytochemically detected in NOD males before (A) and on day 5 (B) and day $8(\mathbf{C})$ post CYP treatment. Pancreata from 4-5 mice per time point were snap-frozen in liquid nitrogen and acetone-fixed. Indirect immunoperoxidase staining using $\mathrm{DAB}$ as substrate was caried out with haematoxylin counterstain. ART2.2-positive cells are marked by a brown reaction deposit. Two replicate experiments gave comparable results. Magnification $\times 200$

cells were polyclonally activated by anti-CD3 $\mathrm{mAb}$ in the absence or presence of NAD, the ART2.2 substrate. For that, we used total spleen cells from ART2.2-expressing B6 and NOD/Lt mice, as well as from NZW/LacJ mice, which do not express ART2.2 due to a defect in their $A r t 2 b$ gene [21]. As shown in Fig 6, inhibitory effects of NAD on CD25 up-regulation were detectable at concentrations as low as $1 \mu \mathrm{mol} / \mathrm{l}$. A concentration higher than $10 \mu \mathrm{mol} / 1$ (resulted in increases $(p<0.01)$ in dead cells as assessed by propidium iodide staining. T cells from ART2.2deficient NZW mice were less sensitive to inhibition by low concentrations of NAD. We then tested the effects of $10 \mu \mathrm{mol} / \mathrm{l} \mathrm{NAD}$ on activation of islet-reactive CD8 T cells isolated from the spleens of NOD.AI4 $\alpha \beta$ Tg mice [14]. The majority (95\%) of CD8 cells in these transgenic mice express the TCR from the islet-derived AI4 CTL clone. AI4 T cells are H2-K ${ }^{\mathrm{d}}$-restricted and recognize an undefined beta-cell antigen; they will lyse ${ }^{51}$ Cr-labeled NOD.scid islet cells [14]. When freshly isolated from spleen and without exposure to their cognate antigen on isolated NOD.scid islets in vitro; these naive NOD.AI4 $\alpha \beta$ Tg T cells expressed ART2.2. When cocultured with NOD.scid target islet cells in the presence or absence of $10 \mu \mathrm{mol} / 1 \mathrm{NAD}, \mathrm{CD} 25$ induction on the CD8 fraction was inhibited $28 \%$ by NAD (data not shown). To test whether NAD suppression of CD25 upregulation retarded the effector function of these CTL, we analy- sed islet cell cytolysis mediated by NOD.AI $4 \alpha \beta \mathrm{Tg}$ $\mathrm{T}$ cells in vitro in the presence of NAD. In this CML assay, splenic AI4 $\mathrm{T}$ cells retained expression of ART2.2 during the $18 \mathrm{~h}$ coculture period with islet cell targets, suggesting that the function of the T cells can be modulated by NAD. When NOD.AI $4 \alpha \beta$ Tg spleen cells were incubated with $\mathrm{Cr}^{51}$-labelled islet cells in the presence of NAD, specific lysis of the islet cells was markedly inhibited at NAD concentrations of $1 \mu \mathrm{mol} / 1$ or more (Fig. 7). Of note, spontaneous lysis of islet cells was not affected by NAD within the tested concentration range, indicating the absence of non-specific NAD cytotoxicity.

CD38 expression on splenic Tcells. The inhibitory effects of NAD on activation and killing in vitro raise the question of whether other NAD-utilizing ectoenzymes might be involved in this process. Using thin layer chromatography to separate products, we found that incubation of NOD/Lt spleen cells with 32P-labelled NAD generated large quantities of ADP-ribose, suggesting that NAD hydrolysis is is a major biochemical event when unsorted populations of splenic leukocytes are studied This could be mediated by CD38, a ubiquitously-expressed NADase that is generally expressed at higher concentrations (mean flourescence intensity) on B lymphocytes than on T cells. We therefore compared the expression of CD38 on T and B lymphocytes from NOD and other mouse strains. No significant difference between NOD and other strains in CD 38 expression on B lymphocytes was observed; however, NOD and NOR showed increased CD38 staining intensities on CD4 and CD8 spleen cells compared to those from BKS, CBA/J, and BALB/cByJ mice (Fig. 8). Thus, expression of CD38 on NOD/Lt T cells was relatively higher than in other unrelated strains. Among the NOD-related strains tested, NON/Lt had the highest proportion of CD38 $8^{\text {high }} \mathrm{T}$ cells, followed by NOR/Lt, with NOD mice having the lowest proportion (data not shown). 


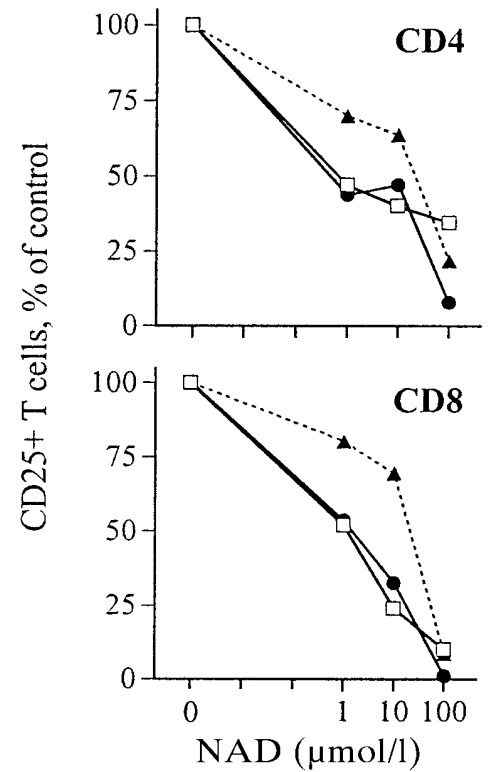

Fig. 6. Differential inhibition of anti-CD3-induced T-cell activation in vitro by NAD in ART2.2-positive vs ART2.2-negative strains. Spleen cells from NOD $(-\square)$, B6 (-) , and NZW (-- -) (ART2.2 null strain) females were activated by $2 \mu \mathrm{g} /$ $\mathrm{ml}$ anti-CD3 $\mathrm{mAb}$ overnight in the presence of the indicated concentrations of NAD in the medium, and CD25 expression was analysed on viable CD4 and CD8 T cells. The profound inhibition of CD25 expression seen in all 3 strains at $100 \mu \mathrm{mol} / 1$ NAD was associated with T-cell toxicity (more than $50 \%$ propidium iodide non-excluding cells). Because various strains expressed different proportions of CD25 + T cells after stimulation in the absence of NAD, these values were taken as respective controls $(100 \%)$ and data are expressed as a relative inhibition. Data are representative of three separate experiments

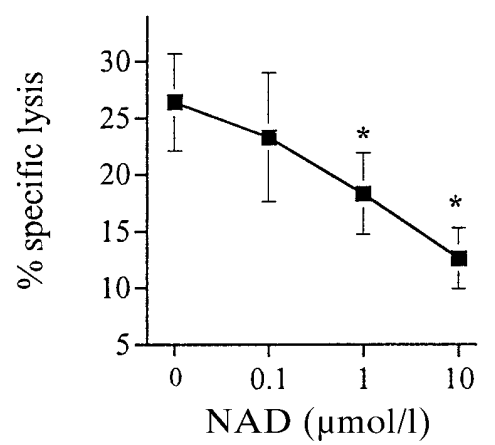

Fig. 7. Inhibition of specific cytotoxicity by NAD. Freshly isolated splenocytes from NOD.AI $\alpha \beta$ Tg mice were co-cultured with dispersed and ${ }^{51} \mathrm{Cr}$ - labelled islet cells overnight with or without NAD. Per cent specific lysis was calculated. Data are means of two experiments done in triplicates. ${ }^{*} p<0.05$ compared to $0 \mu \mathrm{mol} / \mathrm{l}$ NAD (Mann-Whitney two-tailed test)

Mono-ADP ribosylation of $T$ Cells is inhibited in the presence of $B$ lymphocytes and upon T-Cell activation. To test whether CD38 $8^{\text {high }} B$ lymphocytes could affect mono-ADP ribosylation mediated by ART2.2 and others ARTs, we incubated spleen cells with etheno-

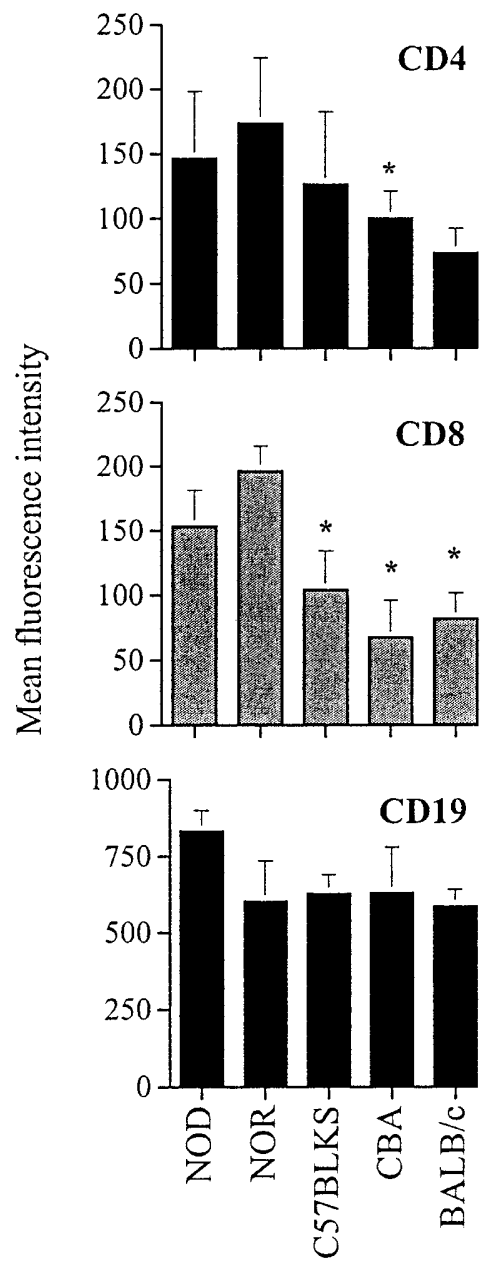

Fig. 8. CD38 expressions on CD4 (upper panel), CD8 (middle panel) and CD19 (lower panel) spleen cells from adult (12-14 wk-old) NOD and NOR mice compared to three other non-related strains. Three males from each strain were used and data are means \pm SE. $* p<0.05$ vs NOD (Student two-tailed $t$ test)

NAD and stained them wit $1 \mathrm{G} 4 \mathrm{mAb}$ which recognizes etheno-ADP-ribosyl moieties [12, 15]. As shown on Figure 9, ADP-ribosylation was more effective in B-lymphocyte-depleted spleen cell preparations (panel $\mathrm{C}$ ). In whole spleen cells containing up to $50 \%$ B lymphocytes, T-cell staining by $1 \mathrm{G} 4$ was reduced, suggesting that $\mathrm{CD} 38^{\text {high }} \mathrm{B}$ lymphocytes hydrolyze etheno-NAD, thereby decreasing availability of the common NAD substrate for ARTs (Fig. 9B). Loss of ART2.2 expression induced by activation with PMA + calcium ionophore was associated with the loss of 1G4 staining [compare high ART2.2 expression (Fig. 9D) and etheno-ADP ribosylation (Fig. 9E) on unactivated T cell subsets versus concomitant loss of ART2.2 and markedly reduced etheno-ADP ribosylation following activation (Fig. 9G and $\mathrm{H}$ respectively)]. These results indicate that other ARTs that might contribute to monoADP ribosylation of T-cell surface proteins are also 

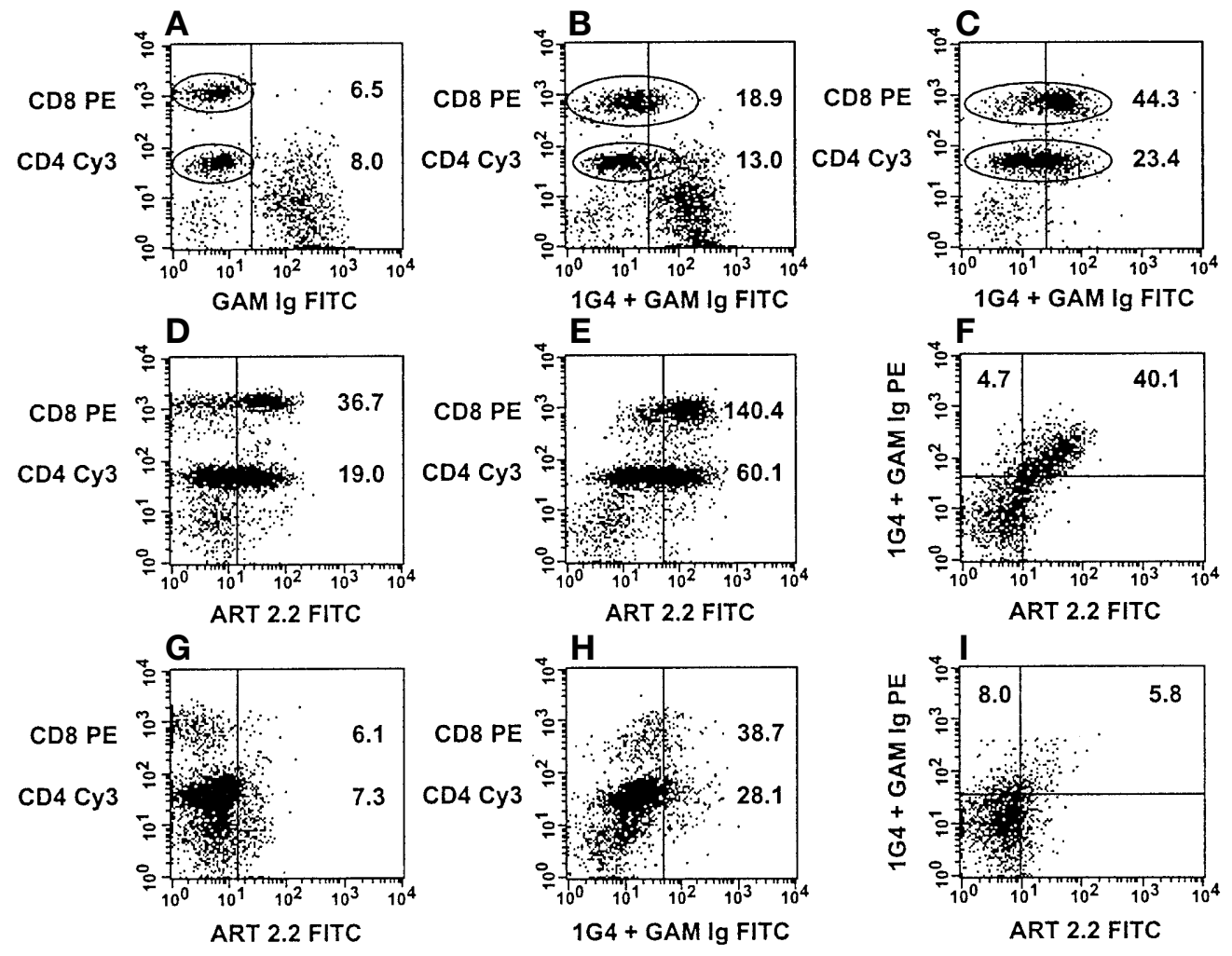

Fig.9A-I. Detection of mono-ADP ribosylation of splenic T cells is influenced by presence of B lymphocytes and T-cell activation state. Whole spleen cells from 12 wk-old NOD male mice $(\mathbf{B})$ or T cells enriched by B-lymphocyte depletion $(\mathbf{C})$ were incubated with etheno-NAD and then stained with 1 G4 mAb plus goat antimouse Ig-FITC (B and C). Staining of the whole spleen with the second $\mathrm{Ab}$ alone $(\mathbf{A})$ is shown for comparison. Panels (D) and (E) show ART2.2 and monoADP ribosylation from ethheno-NAD substrate on unactivated splenic CD4 and CD8 T-cell subsets after B-lymphocyte depletion. Panels $(\mathbf{G})$ and $(\mathbf{H})$ show that loss of ART2.2 staining correlates with marked reduction in etheno-ADP ribosylation in the same B-lymphocyte depleted preparation. Co-staining of T cells for ART2.2 and T-Cell surface etheno-ADP ribosylation in unactivated (F), versus activated (I) T cells in this preparation confirms concomitant loss of both phenotypes after activation (I). Numbers in panels $(\mathbf{A}-\mathbf{C}),(\mathbf{D}),(\mathbf{E}),(\mathbf{G})$ and $(\mathbf{H})$ are mean fluorescence intensities; numbers in panels $(\mathbf{F})$ and (I) indicate percentages of gated populations. Data are representative of three separate experiments. Similar results were obtained with splenic cells from BKS mice

down-regulated (by shedding from the cell surface) upon activation or are minor contributors to auto ADP-ribosylation reactions. The majority of $\mathrm{T}$ cells stained by $1 \mathrm{G} 4$ are ART2.2 $2^{+}$, whereas ART2.2 $2^{-}$cells are unstained by $1 \mathrm{G} 4$ (Fig. 9F and I), suggesting that ARTs preferably modify proteins on the same cell rather than on a neighboring ART-negative one.

\section{Discussion}

It has been hypothesized that ADP-ribosylation of cell surface molecules could be an important means for regulating T-cell functions and that this mechanism could contribute to down-regulating autoimmunity $[1,10,22-24]$. Our study describes the expression of the T-cell ecto-ADP-ribosyltransferase ART2.2 in the NOD/Lt mouse model of Type I diabetes. The pattern of ART2.2-expression in the natural history of Type I diabetes (i.e. in non-manipulated animals) is recapitulated in cyclophosphamide-induced acceleration of this disease. We found the following correlations between disease and the loss of ART2.2 in vivo (1) a delayed ontogeny of ART2.2 expression in the NOD/Lt mouse compared with control mouse strains (2) low ART2.2 expression by islet-infiltrating T cells, and (3) a systemic and lesional loss of ART2.2 after cyclophosphamide treatment (Figs. 4, 5). Together, these findings support the hypothesis that ART2.2 could be a down-regulator of autoimmunity. They provide a valuable background prior to more aggressive strategies aimed at dissecting the role of this enzyme in Type I diabetes; for example the construction of ART2.2 transgenic and ART2.2 knockout animals.

Our results further show that the NOD/Lt model of Type I diabetes differs from the BB-DR rat model in several important respects. Among these differences are the markedly higher numbers of circulating $\mathrm{T}$ cells in NOD and their expression of Art2 gene products (formerly denoted as RT6) [25]. However, 
the delayed ontogeny of $A r t$ gene expression in NOD splenic T cells, previously shown as a reduced transcript abundance by northern analysis [11], and confirmed in our study by flow cytometry using a monoclonal antibody, suggests that this NOD strain-specific characteristic could be one of the many factors rendering this mouse strain singularly susceptible to Type I diabetes. Our finding that CYP-accelerated development of Type I diabetes in NOD males essentially recapitulated the ontogenic pattern of initially reduced ART2.2 expression further suggests that T cells expressing ART2.2 are less pathogenic than those that do not. It should be noted that the developmental delay in ART2.2 expression on splenic T cells from young NOD donors at the peri-weaning period correlates with an NOD strain-specific early expression of IL-2 receptors on CD $4+$ T cells. It is during this time that the first $\mathrm{T}$ cells and their lymphokine transcripts are detected in pancreatic islets [26, 27]. Our finding that naive islet-reactive CTLs from spleens of NOD.AI4Tg mice had reduced killing potency when shown cognate antigen on islet cells in vitro in the presence of NAD, the ART2.2 substrate suggests that ART may not only mark a "resting" or naive phenotype but also participate in its maintenance. Diabetes-resistant BB-DR rats can be rendered diabetic after depleting ART2/RT6-positive $\mathrm{T}$ cells by mAb treatment followed by poly I:C treatment. In preliminary studies, we have failed to accelerate Type I diabetes in 4-week-old NOD/Lt mice using 2 doses of antiART2.2 (Nika 102) mAb. This mAb treatment produced a transient T-lymphopenia for about 20 days but Type I diabetes onset was neither accelerated or retarded. We did not combine this treatment with poly I:C administration as is required for Type I diabetes in non-T-lymphopenic rats [8]. In NOD mice, treatment of prediabetic mice with poly I:C alone suppresses diabetogenesis [28], again highlighting certain essential differences in the rat and mouse models. Although another study using an in vitro model system suggested that CD38, another NAD metabolizing ectoenzyme, identified a regulatory CD4-positive T-cell subset [29], our data showing high CD38 expression on freshly-isolated NOD T cells do not support this.

We have previously shown that resting mouse $\mathrm{T}$ cells shed enzymatically-active ART2.2 antigen upon strong mitogenic activation, rendering them less responsive to auto-ADP ribosylation [12]. Reduced numbers of T-cell expressing surface ART2.2 (as we have observed early in NOD/Lt ontogeny and upon cyclosphosphamide treatment) therefore, might reflect increased shedding of ART2.2. This is of potential interest since the appearance of soluble ART2.2 protein or its activity could represent a useful predictive marker for disease development. Furthermore, shed ART2.2 conceivably could ADP-ribosylate further substrates such as inflammatory mediators or their receptors and thereby contribute to inflamma- tion. Our efforts to quantify soluble ART2.2 or its enyzme activity, in serum for example, have been confounded by a series of factors, including lack of sensitive assay systems, an apparent short half life of ART2.2, and the presence of NADases.

Others have shown that ADP-ribosylation of CTL surface moieties down-regulates T-effector homing and killing functions $[3,10,22,30,31]$. The NZW/ B1nJ mouse does not express ART2.2 due to a mutation in the gene [6]. In this study, we have shown that $\mathrm{NAD}$ at a $1 \mu \mathrm{mol} / \mathrm{l}$ concentration had only a small suppressive effect on activation-induced CD25 expression on NZW/B1nJ $\mathrm{T}$ cells compared to ART2.2-competent NOD or B6 T cells. The toxic effect of increased NAD concentrations on T cells can be explained by ART-mediated auto-ADP-ribosylation of T-cell surface proteins. Conversely, resistance of isolated islet cells to the deleterious effects of this substance can be explained by their apparent lack of ART2-expression. The finding of a gradually increasing damaging effect of NAD on T cells further supports our suggestion that, in an insulitic infiltrate, those T cells still expressing ART2.2 are more likely to autoregulate themselves by ADP-ribosylation of critical signalling molecules on their surfaces than are activated, CD25-positive and ART2.2 negative T cells. A critical issue in this regard is whether sufficient quantities of NAD substrate becomes available to the ART2.2 ectoenzyme at the site of contact with targets. Nicotine adenine dinucleotide is a ubiquitous intracellular molecule involved in mitochondrial electron transport and maintainance of redox potential. Its depletion in beta cells by poly (ADP-ribose) polymerase, or PARP, is considered to be a beta-cytopathic event that can be blocked by nicotinamide [32]. Extracellular NAD concentrations normally fall within the nanomolar - lower micromolar range, but could reach higher concentrations in foci of massive cell death [33]. We have observed that concentrations of NAD above $1 \mu \mathrm{mol} / \mathrm{l}$ will induce apoptosis in non-activated cultures of naive splenic T cells after a $30 \mathrm{~min}$ incubation. Although we have no evidence directly addressing this point, we presume that, in an insulitic lesion, local concentrations of extracellular NAD will increase as more beta cells are lysed.

However, ART is not the only NAD-metabolizing ectoenzyme on leukocytes at the site of inflammation. CD38 is expressed on the cell surfaces of a wide variety of cells, including $B$ and T lymphocytes, NK cells, macrophages, [34] and even on islet cells [35]. It functions mainly as an NAD hydrolase with some ADP-ribosyl cyclase activity. Our data indicate that NOD/Lt and the related NOR/Lt strain express higher concentrations of CD38 on both T and B lymphocytes than do BKS, BALB/cByJ, or $\mathrm{CBA} / \mathrm{J}$ mice. CD38 expression does not depend on the expression of ART2.2, since it is low in both ART2.2 $2^{\text {high }}$ BKS and ART2.2 $2^{\text {low }} \mathrm{CBA} / \mathrm{J}$ strains. On the contrary, 
ART2.2 enzymatic activity is modulated by competition for NAD substrate when B lymphocytes are present. Employing etheno-NAD substrate and the $1 \mathrm{G} 4 \mathrm{mAb}$ to allow detection of etheno-ADP-ribosylated targets on both NOD CD4-positive and CD8positive T-cell subsets, we showed a markedly higher enzymatic activity when B lymphocytes were eliminated. These data provide evidence suggesting competition between ARTs and CD38 for their common NAD substrate. Because CD38, unlike ARTs, has been reported to be up-regulated upon activation [34], its competition with ARTs for the substrate could be even more pronounced on activated than on resting $\mathrm{T}$ cells and thus further impair effective ADP-ribosylation, shifting the balance towards NAD hydrolysis [13].

Acknowledgements. This work was supported by NIH grants DK36175 and DK27722 (to E.H.L.), DFG grant SFB545/B9 (to F. Haag and F. Koch-Nolte), a grant from the Juvenile Diabetes Foundation International and a fellowship from the American Diabetes Association (to V. Ablamunits). Institutional shared services were supported by a National Cancer Institute Cancer Center Support Grant CA34196. We thank Dr. D. Serreze for NOD.AI4 $\alpha \beta$ Tg mice, and S. Langley, W. Schott, B. Regimbal, and P. Jewett for excellent technical assistance. We thank Dr. D. Serreze, D. Roopenian, A. Chervonsky and B. Richards-Smith for reviewing the manuscript.

\section{References}

1. Cetkovic-Cvrlje M, Leiter EH (1997) Mono-ADP Ribosyltransferase Genes and Diabetes in NOD Mice. In: Haag F, Koch-Nolte F (eds): Is There a Relationship? Plenum Press, New York pp. 217-227

2. Koch-Nolte F, Petersen D, Balasubramanian S. et al. (1996) Mouse T cell membrane proteins Rt6-1 and Rt6-2 are arginine/protein mono (ADP-Ribosyl) transferases and share secondary structure motifs with ADP-ribosylating bacterial toxins. J Biol Chem 271: 7686-7693

3. Nemoto E, Yu Y, Dennert G (1996) Cell surface ADP-ribosyltransferase regulates lymphocyte function-associated molecule-1 (LFA-1) function in T cells. J Immunol 157: 3341-3349

4. Zolkiewska A, Okazaki IJ, Moss J (1994) Vertebrate mono-ADP-ribosyltransferases. Mol Cell Biochem 138: 107-112

5. Hollmann C, Haag F, Schlott M. et al. (1996) Molecular characterization of mouse T-cell ecto-ADP-ribosyltransferase Rt6: cloning of a second functional gene and identification of the Rt6 gene-products. Mol Immunol 33: 807-817

6. Koch-Nolte F, Duffy T, Nissen M. et al. (1999) New monoclonal antibody detects a developmentally regulated mouse ecto ADP ribosyltransferase on T cells: subset distribution, inbred strain variation, and modulation by T cell activation. J Immunol 163: 6014-6022

7. Bortell R, Kanaitsuka T, Stevens LA et al. (1999) The RT6 (Art2) family of ADP-ribosyltransferases in rat and mouse. Mol Cell Biochem 193: 61-68

8. Ellerman KE, Richards CA, Guberski DL, Shek WR, Like AA (1996) Kilham rat virus triggers T-cell-dependent autoimmune diabetes in multiple strains of rats. Diabetes 45: $557-562$
9. Greiner D, Malkani S, Doukas J et al. (1997) The T-cell marker RT6 in a rat model of autoimmune diabetes. Adv Exp Med Biol 419: 209-216

10. Okamoto S, Azhipa O, Yu Y, Russo E, Dennert G (1998) Expression of ADP-ribosyltransferase on normal T lymphocytes and effects of nicotinamide adenine dinucleotide on their function. J Immunol 160: 4190-4198

11. Prochazka M, Gaskins HR, Leiter EH, Koch-Nolte F, Haag F, Thiele HG (1991) Chromosomal localization, DNA polymorphism, and expression of Rt-6, the mouse homologue of rat $\mathrm{T}$ cell lymphocyte differentiation marker RT6. Immunogenetics 33: 152-156

12. Kahl S, Nissen M, Girisch R et al. (2000) Metalloproteasemediated shedding of enzymically active mouse ecto ADP-ribosyltransferase ART2.2 upon T cell activation. J Immunol 165: 4463-4469

13. Koch-Nolte F, Abromeit M, Gerber A et al. (2000) Molecular characterization of a family of NAD + metabolizing ectoenzymes in mice and men: endogenous relatives of ADP-ribosylating bacterial toxins. In: Vanduffel L, Lemmens R (eds) Ecto-ATPases and Related Ectonucleotidases. Shaker Publishing, Hamburg pp 221-233

14. Graser RT, DiLorenzo TP, Wang F et al. (2000) Identification of a CD8 $\mathrm{T}$ cell that can independently mediate autoimmune diabetes development in the complete absence of CD4 T cell helper functions. J Immunol 164: 3912-3923

15. Young TL, Santella R (1988) Development of techniques to monitor for exposure to vinyl chloride: monoclonal antibodies to ethenoadenosine and ethenocytidine. Carcinogenesis 9: 589-592

16. Ablamunits V, Elias D, Reshef T, Cohen IR (1998) T cells secreting IFN gamma in NOD mouse diabetes: arrest by p277 peptide treatment. J Autoimmun 11: 73-81

17. Jarpe AJ, Hickman MR, Anderson JT, Winter WE, Peck AB (1991) Flow cytometric enumeration of mononuclear cell populations infiltrating the islets of Langerhans in prediabetic NOD mice: development of a model of autoimmune insulitis for type 1 diabetes. Reg Immunol 3: 305-317

18. Rohane PW, Shimada A, Kim DT et al. (1995) Islet-infiltrating lymphocytes from prediabetic NOD mice rapidly transfer diabetes to NODscid/scid mice.Diabetes 44: $550-554$

19. Charlton B, Bacelj A, Slattery RM, Mandel TE (1989) Cyclophosphamide-induced diabetes in NOD/WEHI mice. Evidence for suppression in spontaneous autoimmune diabetes mellitus. Diabetes 38: 441-447

20. Yasunami R, Bach JF (1988) Anti-suppressor effect of cyclophosphamide on the development of spontaneous diabetes in NOD mice. Eur J Immunol 18: 481-484

21. Koch-Nolte F, Klein J, Hollmann C et al. (1995) Defects in the structure and expression of the genes for the $\mathrm{T}$ cell marker Rt6 in NZW and (NZBxNZW)F1 mice. Int Immunol 7: 883-890

22. Wang J, Nemoto E, Kots AY, Kaslow HR, Dennert G (1994) Regulation of cytotoxic T cells by ectonicotinamide adenine dinucleotide (NAD) correlates with cell surface GPI-anchored/arginine ADP-ribosyltransferase. J Immunol 153: 4048-4058

23. Koch-Nolte F, Haag F, Kastelein R, Bazan F (1996) Uncovered: the family relationship of a T-cellmembrane protein and bacterial toxins.Immunol Today 17: 402-405

24. Haag F, Koch-Nolte F (1998) Endogenous relatives of ADP-ribosylating bacterial toxins in mice and men: potential regulators of immune cell function. J Biol Regul Homeost Agents 12: 53-62

25. Serreze DV, Leiter EH (1995) Insulin Dependent Diabetes Mellitus (IDDM) in NOD Mice and BB Rats: origins in he- 
matopoietic stem cell defects and implications for therapy. In: Shafrir E (ed) Lessons from Animal Diabetes. SmithGordon, London, Vo 5, pp 59-73

26. Miyazaki A, Hanafusa T, Yamada K et al. (1985) Predominance of $\mathrm{T}$ lymphocytes in pancreatic islets and spleen of pre-diabetic non-obese diabetic (NOD) mice: a longitudinal study. Clin Exp Immunol 60: 622-630

27. Fox C, Danska J (1997) IL-4 expression at the onset of islet inflammation predicts nondestructive insulitis in Nonobese Diabetic mice. J Immunol 158: 2414-2424

28. Serreze DV, Hamaguchi K, Leiter EH (1989) Immunostimulation circumvents diabetes in NOD/Lt mice. J Autoimmun 2: 759-776

29. Read S, Mauze S, Asseman C, Bean A, Coffman R, Powrie F (1998) CD38 + CD45RB(low) CD4 + T cells: a population of $\mathrm{T}$ cells with immune regulatory activities in vitro. Eur J Immunol 28: 3435-3447

30. Wang J, Nemoto E, Dennert G (1996) Regulation of CTL by ecto-nicotinamide adenine dinucleotide (NAD) involves ADP-Ribosylation of a $5^{1} 6^{1 \mathrm{ck}}$ associated protein. J Immunol 156: 2819-2827

31. Liu Z-X, Yu Y, Dennert G (1999) A cell surface ADP-ribosyltransferase modulates $\mathrm{T}$ cell receptor association and signaling. J Biol Chem 274: 17399-17401

32. Kolb H, Burkart V (1999) Nicotinamide in type 1 diabetes. Mechanism of action revisited. Diabetes Care 22 [Suppl 2]: B16-B20

33. Zupo S, Rugari E, Dono M, Taborelli G, Malavasi F, Ferrarini M (1994) CD38 signaling by agonistic monoclonal antibody prevents apoptosis of human germinal center B lymphocytes. Eur J Immunol 24: 1218-1222

34. Lund F, Solvason N, Grimaldi JC, Parkhouse RME, Howard M (1995) Murine CD38: an immunoregulatory ectoenzyme. Immunol Today 16: 469-473

35. Kato I, Yamamoto Y, Fujimura M, Noguchi N, Takasawa S, Okamoto H (1999) CD38 disruption impairs glucose-induced increases in cyclic ADP-ribose, $[\mathrm{Ca} 2+] \mathrm{i}$, and insulin secretion. J Biol Chem 27: 1869-1872

36. Cockayne DA, Muchamuel T, Grimaldi JC et al. (1998) Mice deficient for the ecto-nicotinamide adenine dinucleotide glycohydrolase CD38 exhibit altered humoral immune responses. Blood 92: 1324-1333

37. Signore A, Cooke A, Pozzilli P, Butcher G, Simpson E, Beverly PCL (1987) Class II and IL2 receptor positive cells in the pancreas of NOD mice. Diabetologia 30: 902-905

38. Grimaldi JC, Balasubramanian S, Kabra NH et al. (1995) CD38-mediated ribosylation of proteins. J Immunol 155: 811-817

39. Serreze DV, Leiter EH (1988) Defective activation of T suppressor cell function in Nonobese Diabetic mice. Potential relation to cytokine deficiencies. J Immunol 140: 3801-3807

40. Zocchi E, Usai C, Guida L et al. (1999) Ligand-induced internalization of CD38 results in intracellular $\mathrm{Ca} 2+$ mobilization: role of NAD + transport across cell membranes. FASEB J 13: 273-283

41. Serreze DV, Prochazka M, Reifsnyder PC, Bridgett MM, Leiter EH (1994) Use of recombinant congenic and con- genic strains of NOD mice to identify a new insulin dependent diabetes resistance gene. J Exp Med 180: 1553-1558

42. Han MK, Cho YS, Kim YS, Yim CY, Kim UH (2000) Interaction of two classes of ADP-Ribose transfer reactions in immune signaling. J Biol Chem 275: 20799-20805

43. Shimada A, Rohane P, Fathman CG, Charlton B (1996) Pathogenic and protective roles of CD45RB(low) CD4 $(+)$ cells correlate with cytokine profiles in the spontaneously autoimmune diabetic mouse. Diabetes 45: 71-78

44. Baxter AG, Kinder SJ, Hammond KJL, Scollary R, Godfrey DI (1997) Association between alpha-beta$\mathrm{TCR}^{+} \mathrm{CD} 4{ }^{-C D} 8-\mathrm{T}$-cell deficiency and IDDM in NOD/Lt mice. Diabetes 46: 572-582

45. Herbelin A, Gombert HM, Chatenoud L, Lepault F, Bach JF (1998) Mature mainstream TCR alpha beta + CD4 + thymocytes expressing L-selectin mediate active tolerance in the nonobese diabetic mouse. J Immunol 161: 2620-2628

46. Pankewycz O, Strom TB, Rubin-Kelley VE (1991) Isletinfiltrating T-cell clones from nonobese diabetic mice that promote or prevent accelerated onset diabetes. Eur J Immunol 21: 873-879

47. Rabinovitch A (1998) An update on cytokines in the pathogenesis of insulin-dependent diabetes mellitus. Diabetes Metab Rev 14: 129-151

48. Feili-Hariri M, Frantz MO, Morell PA (2000) Prevention of diabetes in the NOD mouse by a Thl clone specific for a hsp60 peptide. J Autoimmun 14: 133-142

49. Elias D, Tikochinski Y, Frankel G, Cohen IR (1999) Regulation of NOD mouse autoimmune diabetes by T cells that recognize a TCR CDR3 peptide. Int Immunol 11: 957-966

50. Akhtar I, Gold JP, Pan LY et al. (1995) LCD4( + ) beta islet cell-reactive $\mathrm{T}$ cell clones that suppress autoimmune diabetes in nonobese diabetic mice. J Exp Med 182: 87-97

51. Ablamunits V, Elias D, Cohen IR (1999) The pathogenicity of islet-infiltrating lymphocytes in the non-obese diabetic (NOD) mouse. Clin Exp Immunol 115: 260-267

52. Salomon B, Lenschow DJ, Rhee L et al. (2000) B7/CD28 costimulation is essential for the homeostasis of the CD4 + CD25 + immunoregulatory T cells that control autoimmune diabetes. Immunity 12: 431-440

53. Shevach EM (2000) Regulatory T cells in autoimmmunity. Annu rev Immunol 18: 423-449

54. Haag F, Koch-Nolte F, Kuhl M, Lorenzen S, Thiele H (1994) Premature stop codons inactivate the RT6 genes of the human and chimpanzee species. J Mol Biol 243: $537-546$

55. Koch-Nolte F, Haag F, Braren R et al. (1997) Two novel human members of an emerging mammalian gene family related to mono-ADP-ribosylating bacterial toxins. Gemomics 39: 370-376

56. Frye RA (1999) Characterization of five human cDNAs with homology to the yeast SIR2 gene: Sir2-like proteins (sirtuins) metabolize NAD and may have protein ADP-ribosyltransferase activity. Biochem Biophys Res Commun 260: $273-279$ 